Journal of Neurology, Neurosurgery, and Psychiatry 1987;50:801-803

Short report

\title{
Tardive dystonia in a psychiatric hospital
}

\author{
JOSEPH H FRIEDMAN,* L THOMAS KUCHARSKI, $†$ RICHARD L WAGNER $\dagger$ \\ From the Division of Neurology, Department of Medicine, Roger Williams General Hospital,* RI \\ Neuropsychiatric Research and Training Program, $\nmid$ Cranston, and Butler Hospital, Department of Psychiatry, \\ Providence, RI, USA
}

SUMMARY Tardive dystonia is a relatively uncommon long term complication of neuroleptic therapy. The prevalence of this syndrome is unknown. This study, of chronic hospitalised psychiatric patients, revealed a tardive dystonia prevalence of $1.5 \%$. Risk factors in this small population appeared to be mental retardation and convulsive therapy

Neuroleptic medications have been implicated in a wide variety of movement disorders. Early adverse reactions include acute dystonia, Parkinsonism and akathisia. Complications occurring after prolonged neuroleptic exposure, (usually years, but occasionally after a few months ${ }^{12}$ ) are termed "tardive" movement disorders. The most common and most frequently recognised are the oral, lingual, buccal movements termed tardive dyskinesias. The prevalence of these movements in the neuroleptic treated population varies from $0.5 \%$ to $51.6 \%$, depending on the study with an average of $20 \% .^{3}$ Less frequently described is tardive dystonia.

Tardive dystonia is defined as a chronic dystonia, occurring for the first time after a minimum of 2 months of neuroleptic use in a patient without another explanation (such as stroke, Huntington's disease, Parkinson's disease, Wilson's disease) who has a negative family history for the disorder. ${ }^{1}$ It is frequently present with the more common, oralbuccal-lingual movements of tardive dyskinesia. ${ }^{14}$

Although many studies have been performed analysing prevalence rates and risk factors for tardive dyskinesia $^{3}$ no published study has focused on the prevalence and risk factors for dystonia. Owens et $\mathrm{al}^{5}$ in a survey of a psychiatric hospital found a prevalence of $2.2 \%$ of retrocollis or torticollis and $2.7 \%$ of truncal torsion movements in a psychiatric hospital but gave no further description. No other dystonias were noted and the possibility of some of these cases being acute dystonic reactions must be considered.

We report the findings of our survey of patients of a total of 331 patients, housed in September, 1984, in the Institute for Mental Health (IMH), the only public supported psychiatric hospital in Rhode Island. This is the first report of the prevalence of tardive dystonia in any population.

\section{Subjects}

The patients housed in the IMH, are similar to those in most public psychiatric hospitals. Patients suffering from Alzheimer's disease, moderate to severe retardation or who are outside the age range of 18-70 years are hospitalised elsewhere. The policy regarding patients with other organic neurological disorders which include behaviour disorders is less clear, so that some patients have had strokes, subarachnoid haemorrhages, hypoglycaemic encephalopathy or head trauma. These patients, with organic disorders represent however a small minority of the population. All have behavioural abnormalities. Aside from this mixture of "organic" and "non-organic" cases, there is also a mixture of acutely, chronically, and intermittently hospitalised patients. These patients are unable, for psychiatric or social reasons, to be maintained outside the hospital. The acutely hospitalised patients generally lack health insurance. By and large, these patients are poorly educated and come from low income families. Complete histories are not available on all patients but all are believed to have had neuroleptic exposure.

\section{Methods}

All patients in the Institute for Mental Health were asked to give informed consent for a brief examination to identify movement disorders. Three hundred and thirty-one of 352 residents of the IMH in September 1984 were evaluated by a neurologist (JHF) and a clinical psychologist (LTK), each with expertise in drug induced movement abnormalities. All patients were rated on the Abnormal Involuntary Movement Scale ${ }^{6}$ [AIMS] and the Reversible Extrapyramidal Symptom Scale ${ }^{7}$ [REPS]. Subjects were examined between 8:30 am and noon, after the first morning dose of all medications were withheld. Patients initially identified as having significant dystonia (greater than or equal to 2 on the REPS item \#6) were re-evaluated by the neurologist approximately 6 and 9 months after the initial evaluation. These later evaluations were performed in the morning but without alteration of the usual medication regimen. 


\section{Results}

Five patients who were identified on initial examination to be dystonic still had evidence of dystonia on re-examination at 6 months. Four still had dystonia at one year. One patient who had severe dystonia at 8 months died before the ninth month. He had eloped and was found dead. No necropsy was performed and the cause of death is unknown. One case of dystonia, the only female and the only case not involving the neck or face, resolved on re-examination at 9 months after a change of medication.

While the number of cases is small, representing $1.5 \%$ (five affected of 331 screened) of the population, the fact that mostly males suffered from tardive dystonia is notable. The make-up of the studied population was 174 males and 157 females. It was not possible, owing to incomplete records, to determine when the dystonia began. In no case was the dystonia thought to warrant any special treatment by the staff prior to its being identified in this survey. Only one patient has had an evaluation for Wilson's disease, with serum ceruloplasmin, liver function tests and a slit lamp examination but no patient has any known family member with any neurological disorder. All patients have had multiple normal liver function tests on blood studies. None have Kayser-Fleischer rings on examination with an opthalmoscope. Unusual features of this group of patients included: two of the four men have had psychotic states that have not been controlled on trials of various medications and different dosage regimens. This lack of control has been evident for several years in one patient, and for the one year the other has been at the IMH. Three of the four men had subnormal intellects as measured by intelligence tests, when the patients were in their teens or early twenties, before any movement disorder was present. One intellectually subnormal patient has a dysmorphic face. All but one had evidence of typical oral-buccal-lingual dyskinesias, but none had focal neurological abnormalities other than their dystonias. Two of the men had received courses of ECT. One of these had also been treated with an insulin "subcoma". The dystonias were quite obvious in the four men, but were not so severe as to be debilitating. In no case was walking significantly affected. Three of the four men had dystonias involving the neck. All had been on multiple neuroleptic agents over many years. This fact did not distinguish them from many other patients at the IMH. Their medication doses were comparable at the time of this study to the rest of the IMH in chlorpromazine equivalents. ${ }^{8}$

\section{Discussion}

In this small study we have determined a prevalence of tardive dystonia of $1.5 \%$. The results from our method of withholding the first daily dose of medication is obviously confounded by the variable pharmacodynamics of the different drugs used. Patients on long acting depot medications clearly were not influenced by this tactic. Other patients, whose doses may have been given unevenly during the day, clearly were subject to different stresses and pharmacological changes. While some of these unavoidable deficits were corrected by repeated evaluations at random times during the normal workday without alterations in drug regimen, patients initially missed on the first screening were not re-evaluated. Therefore this estimate is clearly an under-reporting.

Another problem with this study is that none of these patients has had a complete battery of tests including CT scan, serum ceruloplasmin and slit lamp exam of the eyes. Although Wilson's disease may develop late in life, ${ }^{9}$ it is unlikely (although not impossible) without a family history for this or other diseases to have been overlooked. Because this study was specifically designed to find dystonia, milder cases might have been picked up than would "ordinarily" be the case in a tardive dyskinesia screening. Only one case whose dystonia resolved would fall into this category.

Follow up one year after initial evaluation revealed significant changes in the movements of three patients. One had marked resolution of torticollis but newly developed blepharospasm and akathisia. One had marked worsening of blepharospasm and akathisia. The one female improved.

Certain features of these cases match well with other reports in the literature on tardive dystonia. Specifically, Burke et al ${ }^{1}$ reported that in the 17 patients for whom the developmental history was known, seven had abnormalities. Gimenez-Roldan et $a l^{4}$ reported nine patients with tardive dystonia in whom three had mild retardation and a fourth had had an extensive frontal leukotomy. A high percentage of patients described in case reports also suffered from organic impairments. ${ }^{10-15}$ The percentage of cases of tardive dystonia though with either a history of ECT or some organic impairment, as demonstrated by delayed developmental milestones or retardation is quite striking when compared to patients with tardive dyskinesia, in whom clear evidence of central nervous system dysfunction has not clearly been implicated as a risk factor. ${ }^{3}$

The prevalence of dystonia varies with ethnic origin. In the US population as a whole, the frequency is estimated at 0.3 per $100,000^{16}$ making our prevalence of $1.5 \%$ unexpectedly high.

We conclude, from our study and the several reports in the literature that tardive dystonia, secondary to neuroleptic medications exists, that it has a 
prevalence in a chronic care psychiatric population of $1-2 \%$, and that mental retardation and ECT appear to be risk factors for its development.

We thank RE Burke, MD for helpful advice, and C Hopp for manuscript preparation.

\section{References}

1 Burke RE, Fahn S, Jankovic J, et al. Tardive dystonia: late-onset and persistent dystonia caused by antipsychotic drugs. Neurology 1982;32:1335-46.

2 Tarsy D. History and definition of tardive dyskinesia. $J$ Clin Neuropharmacol 1983;6:91-99.

3 Kane JM, Smith JM. Tardive dyskinesia, prevalence and risk factors, 1959 to 1979. Arch Gen Psychiatry 1982; 39:473-81.

4 Gimenez-Roldan S, Mateo D, Bartolome P. Tardive dystonia and severe tardive dyskinesia. A comparison of risk factors to prognosis. Acta Psychiatrica Scand 1985;71:488-94.

5 Owens DGC, Johnstone EC, Frith CD. Spontaneous involuntary disorders of movement. Their prevalence, severity and distribution in chronic schizophrenia with and without treatment with neuroleptics. Arch Gen Psychiatry 1982;39:452-61.

6 Chien CP, Mascio A, Cole JO: Antiparkinson agents and depot phenothiazines. Am J Psychiatry 1974;131:86-90.
7 Guy W. ECDEU Assessment Manual for Psychopharmacology, Department of Health, Education and Welfare, Washington DC 1976.

8 Wyatt RS. Biochemistry and Schizophrenia (Part IV). The neuroleptics - their mechanism of action: a review of the biochemical literature. Psychopharmacol Bull 1976;12:5-50.

9 Strickland GT, Leu ML. Wilson's disease: Clinical and laboratory manifestations in 40 patients. Medicine 1975;54:113-138.

10 Druckman R, Seelinger D, Thulin B. Chronic involuntary movements induced by phenothiazines. $J$ Nerv Ment Dis 1962;135:69-76.

11 Angle CR, McIntire MS. Persistent dystonia in a brain damaged child after ingestion of a phenothiazine. $J$ Pediatr 1968;78:124-6.

12 Harenko A. Retrocollis as an irreversible late complication of neuroleptic medication. Acta Neurol Scand 1967;43 (Suppl 31): 145-6.

13 Weiner WJ, Warner TR: Mania-induced remission of tardive dyskinesia in manic depressive illness. $\mathrm{Ann} \mathrm{Neu-}$ rol 1982;12:229-30.

14 Keegan DL, Rajput AH. Drug induced dystonia tarda: treatment with levodopa. Disease of the Nervous System 1973;34:167-9.

15 Crane GE. Persistent dyskinesia. Br J Psychiatry 1973;122:395-405.

16 Eldrige $R$. The torsion dystonias: literature review and genetic and clinical studies. Neurology 1970;11:1-78. 